\title{
LA ENSEÑANZA DE LA FÍSICA EN LOS CURRICULOS DE INGENIERÍA
}

\section{THE TEACHING OF PHYSICS IN ENGINEERING CURRICULA}

\author{
Julio Enrique Duarte ${ }^{1}$ \\ Faustino Reyes Caballero ${ }^{2}$ \\ Flavio Humberto Fernández Morales ${ }^{3}$
}

\section{Resumen}

En este trabajo presentamos nuestras reflexiones sobre la enseñanza de la Física en los currículos de ingeniería. En conclusión, pensamos que aun con los limitantes de espacio y tiempo, se presenta un micro-diseño de los cursos de Física Básica que deben incluirse en los currículos de Ingeniería, teniendo en cuenta aspectos de motivación, contenidoy programación.

Palabras clave: Enseñanza de la Física, Currículos de Ingeniería.

\section{Abstract}

Recibido: Agosto 17 de 2013 Aceptado: Diciembre 06 de 2013

In this paper we present our reflections on the teaching of physics in engineering curricula. In conclusion, despite of the limitations of space and time, a micro-design of the basic physics courses to be included in the curricula of engineering were presented, considering aspects as motivation, content and programming.

Key words: Teaching of Physics, Curricula of Engineering.

\footnotetext{
1 Doctor en Ciencias Físicas, Profesor Titular, Universidad Pedagógica y Tecnológica de Colombia. Grupo de Energía y Aplicación de Nuevas Tecnologías. E-mail: julioenriqued1@gmail.com

2 Doctor en Ciencias Física, Profesor de Física, Escuela de Ingeniería Electrónica, Universidad Pedagógica y Tecnológica de Colombia. Grupo de Energía y Aplicación de Nuevas Tecnologías. E-mail: carefa4@hotmail.com

3 Doctor en Ingeniería Electrónica, Profesor Titular, Universidad Pedagógica y Tecnológica de Colombia. Grupo de Energía y Aplicación de Nuevas Tecnologías.E-mail:flaviofm1@gmail.com
} 


\section{Introducción}

Los estudiantes, docentes y directivos deben ser conscientes del papel social que juega el Ingeniero y de la importancia de la Física en su formación al igual que en su desempeño profesional. Los Ingenieros son personas que aplican teorías y principios de las ciencias y las matemáticas para investigar y desarrollar soluciones económicas a problemas técnicos. Su trabajo es el nexo entre las necesidades sociales percibidas y las aplicaciones comerciales, se dedican a hacer que las cosas funcionen de forma más eficaz y eficiente (TryEngineering, 2014).

Es misión de la Física aprender modos de pensar y actuar que sean efectivos para describir y predecir el comportamiento del mundo real. El conocimiento científico, no se muestra directa y llanamente a nuestra percepción, debe ser buscado, encontrado por medio de un trabajo indagatorio (Freeland, 1984; Sabino, 1994). Así, la Física debe ser vista como una de las actividades que el hombre realiza, como un conjunto de acciones encaminadas y dirigidas hacia un determinado fin, que no es otro que el obtener un conocimiento verificable sobre los fenómenos de la naturaleza. Indudablemente, en cuanto a sus propósitos y medios, esta actividad tiene fundamentales diferencias con respecto a los sistemas religiosos y filosóficos, al pensamiento mágico, al empirismo físico, y a otras manifestaciones de la actividad humana. Resulta importante resaltar aquí, particularmente a los estudiantes, que no debemos confundir una afirmación respecto a un objeto o hecho, que puede ser cierta o falsa, con el proceso mediante el cual se ha obtenido el conocimiento cuyo resultado es esa afirmación.

La Física es el campo de conocimiento científico de más rápido desarrollo que existe. No existe un conjunto limitado de leyes fundamentales que podamos resumir en una página y con ellas mostrar cómo operan en toda las circunstancias posibles. Aun no conocemos todas las leyes fundamentales. Cada parte del todo del mundo real que hoy conocemos es solo una cierta forma de aproximación a la verdad completa o la verdad completa hasta donde la conocemos.

La Física incluye tanto conocimiento, que resultaría absurdo decir que un estudiante de ingeniería pueda lograr aprender durante su formación de pregrado. El presente trabajo nos permite poner nuestras reflexiones sobre la enseñanza de la Física en los currículos de ingeniería, al alcance de la comunidad universitaria: profesores, estudiantes y directivos, particularmente de la Universidad Pedagógica y Tecnológica de Colombia. En conclusión, pensamos que aún con los limitantes de espacio y tiempo, se presenta un micro-diseño de los cursos de Física Básica que harían parte de los currículos de Ingeniería, incluyendo aspectos de motivación, contenido y programación. Se trata de que los estudiantes de ingeniería comprendan la Física, aprendan del papel significativo que juega la Física en la cultura de nuestros tiempos.

\section{Propuesta de cursos de física básica para un pensum académico en ingeniería}

La comprensión de la Física requiere de ciertos aspectos fundamentales que claramente deben ser tenidos en cuenta: a) despertando interés en la búsqueda de respuestas; b) familiaridad y competencia en labores de observación y experimentación; c)análisis históricos de los mecanismos de creación y confrontación de ideas; d) reconocimiento de la forma como se estructura la Física y su lenguaje; e) conocimiento y habilidad en el empleo de métodos Físico matemáticos; y e) la proyección social del conocimiento científico y tecnológico. El presente listado de cursos procura resaltar estos aspectos fundamentales. La tabla 1 presenta una sugerencia para la programación de los cursos de Física Básica.

\subsection{Física Experimental I}

Temas: Física: la ciencia fundamental del mundo 
escolasticismo dominaron el pensamiento humano durante siglos. Los interrogantes acerca del mundo natural se respondían mediante argumentos dialécticos (es decir, simplemente hablando) y no se hacía intento alguno para verificar sus afirmaciones.

Aristóteles, comparando la caída libre de dos cuerpos de igual forma y materia específica, sostuvo que el cuerpo más pesado cae más rápido que el más ligero, concepto equivocado que se aceptó como norma durante aproximadamente 1800 años hasta que Galileo llevo a cabo la socialización de su experimento al dejar caer dos objetos de igual forma y materia específica, uno liviano y el otro pesado, desde lo alto de la torre inclinada de Pisa. Mostrando que, luego de numerosos estudios con el péndulo, la caída libre de los cuerpos es independiente de la masa; e igualmente, estableciendo así, el principio más fundamental de la Física: la comprobación de todo conocimiento es el experimento. El experimento es el único juez de la verdad científica.

Los experimentos de Galileo con respecto al péndulo simple y al movimiento en el plano inclinado, son referidos una y otra vez por las autoridades científicas a ser usados para orientar la enseñanza de la Física Experimental Básica. Estos experimentos son sumamente sencillos de realizar con material simplísimo. Sean estos los experimentos escogidos o no, lo importante es que el estudiante desarrolle su trabajo experimental con un espíritu análogo al del investigador original; es decir, tratando de aprender mientras se experimenta. Esto puede ser fácil de decir y de comprender, pero lo más importante es que se haga. Aún en muchos lugares se enseña mal la Física Experimental Básica, aunque se disponga de variado y rico material de experimentación.

En la tabla 2 se presentan los tres eventos que deben caracterizar el proceso de enseñanzaaprendizaje de los cursos introductorios de Física Experimental Básica. En el trabajo experimental no

\begin{abstract}
INICIO
El estudiante aísla, del complejo de los fenómenos naturales, algún caso fácil de observar repetidas veces; por ejemplo, el fenómeno del péndulo simple. El estudiante debe partir de los aspectos y procesos de la realidad más sencillos para poderlos analizar como es debido.

\begin{tabular}{l} 
DESARROLLO \\
\hline (a) El estudiante examina ciertas ideas - hipótesis acerca del procedimiento \\
correcto a seguir con sus mediciones y de los resultados que se espera \\
obtener. Las hipótesis son conjeturas a ser confirmadas, refutadas o \\
modificadas, mediante lo cual la experiencia se expande. \\
(b) El estudiante establece conceptos y definiciones operacionales (por \\
ejemplo, define los parámetros: periodo, frecuencia, longitud del péndulo y \\
la masa que cuelga del mismo). \\
(c) El estudiante realiza una serie de operaciones manuales para lograr su \\
objetivo, y sigue activamente la naturaleza con sus cuestionamientos. \\
(d) El estudiante establece procesos de acumulación y clasificación de \\
datos. \\
(e) El estudiante establece la existencia de leyes empíricas, es decir, \\
relaciones entre dos o más de los parámetros mencionados.
\end{tabular}
\end{abstract}
FINAL

El estudiante reporta sus resultados: nota de avance, cartelera, poster o artículo (paper), es decir, aprende a socializar el resultado de sus trabajos.

Tabla 2. Metodología de los cursos introductorios de Física Experimental Básica

solo hay que observar y tomar bien las medidas, sino que es preciso recolectarlas ordenadamente y luego analizarlas para descubrir relaciones matemáticas entre las variables medidas. Algunas técnicas sencillas de linealización de curvas y construcción de gráficas con escalas logarítmica y semilogarítmica ayudan mucho en este trabajo. No se debe pensar que el análisis de datos experimentales es un ejercicio trivial. Es muy válido que se incluya todo tipo de herramientas de programación actuales disponibles (software: excel, matlab, origin); e igualmente, tanto para los cursos experimentales como en los teóricos, desarrollar prácticas del empleo de métodos numéricos - programación en el modelado y simulación de procesos, por ejemplo, el sistema solar.

\subsection{Mecánica de la Partícula}

Temas: La descripción del movimiento. Leyes de Newton. Naturaleza de las fuerzas: Fuerzas 


\begin{tabular}{|c|c|c|}
\hline Parámetro/Ley & Forma diferencial & Forma integral \\
\hline Posición & $\overrightarrow{r(t)}=x(t) \hat{t}+y(t) f+z(t) \hat{k}$ & $\overrightarrow{r(t)}=\int \overrightarrow{v(t)} d t+\overrightarrow{C_{2}}$ \\
\hline Velocidad & $\overrightarrow{v(t)}=\frac{\overrightarrow{d r(t)}}{d t}=\frac{d x(t)}{d t} \hat{\imath}+\frac{d y(t)}{d t} \hat{j}+\frac{d z(t)}{d t} \hat{k}$ & $\overrightarrow{v(t)}=\int \overrightarrow{a(t)} d t+\overrightarrow{C_{1}}$ \\
\hline Aceleración & $\overrightarrow{a(t)}=\frac{d \vec{x}(t)}{d t}=\frac{d v_{x}(t)}{d t} \hat{i}+\frac{d w_{y}(t)}{d t} \hat{j}+\frac{d v_{z}(t)}{d t} \hat{k}$ & $m(t)=m ; \overrightarrow{a(t)}=\frac{\sum F_{\text {ext }}}{m}$ \\
\hline \multicolumn{3}{|c|}{ Segunda Ley de Newton } \\
\hline \multicolumn{3}{|c|}{$\sum \overrightarrow{F_{e x t}}=\frac{\overrightarrow{d P(t)}}{d t}=\frac{d}{d t} m(t) \overrightarrow{v(t)}=m(t) \frac{\overrightarrow{v(t)}}{d t}+\overrightarrow{v(t)} \frac{d m(t)}{d t}$} \\
\hline & $\vec{F}=\mathrm{G} \frac{\mathrm{Mm}}{\mathrm{r}^{2}} \hat{p}$ & \\
\hline
\end{tabular}

Tabla 3. Formulación de la mecánica Newtoniana

fundamentales de la naturaleza, Fuerzas de contacto y Fuerzas ficticias. Conservación de la cantidad de movimiento lineal, impulso, centro de masa y colisiones. Conservación de la cantidad de movimiento angular, momento de fuerza. Conservación de la energía mecánica, trabajo y potencia.

Justificación: La experimentación ayuda a obtener las leyes empíricas básicas; sin embargo, se requiere del razonamiento, la imaginación, la creatividad, para establecer la base conceptual de estas sugerencias, explicar lo que hay detrás de todas ellas, hacer las grandes generalizaciones: $¡ \mathrm{Es}$ necesario construir una teoría!. El propósito de una teoría física es conectar coherentemente, mediante una estructura lógico-matemática, el mayor número de sucesos observables diferentes a partir de un número de conceptos y postulados ó principios generales.

La Física Matemática constituye un lenguaje de alta precisión y de extraordinario poder de síntesis, en ella los símbolos matemáticos adquieren significado físico, mediante definiciones operacionales se indica qué operaciones y mediciones físicas deben efectuarse en cada caso para determinar sus respectivos valores numéricos. Las definiciones operacionales son el medio de establecer, de la mejor manera posible, la confrontación entre una teoría y los resultados experimentales. Por coherente que sea la parte matemática de una teoría, el valor que pueda tener para describir y explicar una parte del mundo real solamente lo determina la experimentación.

Isaac Newton desarrolló una precisa y poderosa teoría sobre el movimiento. La mecánica Newtoniana representa en la historia de las ciencias el reemplazo de un esquema meramente descriptivo de los fenómenos a un esquema racional (lógico-matemático). Partiendo de las definiciones de velocidad y aceleración y de las leyes particulares sobre movimiento dadas por Galileo, Newton ideó otras definiciones operacionales complementarias esenciales para la descripción del movimiento. En la tabla 3 son presentadas las ecuaciones que gobiernan el movimiento de traslación de una partícula en el espacio tridimensional. Dado que en tiempos de Newton se carecía de los métodos matemáticos necesarios, éste debió desarrollar su propia matemática, al hacerlo sentó las bases de lo que hoy se conoce como Cálculo, rama de la matemática absolutamente imprescindible en el estudio de todas las ciencias e ingenierías.

La teoría de Newton expresa que los cambios de movimiento de cualquier objeto son el resultado 
de las acciones externas (interacciones o fuerzas) que actúan sobre él. El mayor triunfo de la mecánica clásica fue el éxito del propio Newton al analizar la forma de comportarse el sistema solar, con su concepto de fuerza como la causa de los cambios de movimiento y con su postulado de una ley particular de las fuerzas, la ley de la fuerza de gravitación que variaba inversamente con el cuadrado de la distancia. En la tabla 3 se presenta la formulación más general de la Segunda Ley de Newton, en términos de la cantidad de movimiento lineal y la formulación de la fuerza de atracción gravitacional. La mecánica newtoniana explica el movimiento de los cuerpos, tal como el de cualquier objeto de uso cotidiano que cae; un proyectil disparado en una cierta dirección y con un ángulo dado; el de las distintas partes de una máquina; el de la Luna, etc.

El curso se fundamenta en el modelo de partícula (corpúsculo o punto material). En sentido riguroso, no hay nada en la naturaleza que cumpla esta definición. Se considera partícula un objeto cuyas dimensiones pueden despreciarse cuando se describe su movimiento. Por supuesto, esta posibilidad depende de las condiciones concretas del problema. Así, se pueden considerar los planetas como puntos materiales al estudiar su movimiento alrededor del Sol; pero no, evidentemente, cuando se considera su rotación alrededor de sus ejes. Un modelo es un ensayo de la realidad. Ensayando modelos el científico práctica encontrase con la realidad del mundo real. Al construir un modelo, el científico puede reducir un objeto, un sistema o una teoría a una forma manejable.

Cuando nos preguntamos sobre la naturaleza de las fuerzas, debemos conducirnos necesariamente fuera del campo de los fenómenos mecánicos. Durante los últimos cien años, los físicos han acumulado un número de pruebas cada vez mayor de que todas las interacciones entre objetos o materiales distintos, así como cualquiera de las interacciones que por millones y millones se observan a diario, se pueden reducir a combinaciones de cuatro fuerzas fundamentales: La fuerza de la gravedad, la fuerza electromagnética, la fuerza nuclear débil y la fuerza nuclear fuerte (Greene, 2002). Esta excursión actualizada sobre el conocimiento "nuevo" del mundo físico resulta excitante a los estudiantes y es un ingrediente importante del papel cultural de la Física.

Entre la gran variedad y complejidad de los movimientos que suelen ejecutar los cuerpos, tales como traslación, rotación y oscilación, se descubren ciertos aspectos relativamente simples. Con la ayuda de las leyes de Newton, se descubre que en todos estos diversos movimientos hay algunas propiedades que permanecen invariables y es posible aislar algunas nuevas magnitudes cuyo valor se conserva mientras todo lo demás cambia. Igualmente, las leyes de conservación nos pueden conducir eventualmente fuera del campo de los fenómenos mecánicos. Dado que, por ejemplo, podemos transformar energía mecánica en energía eléctrica, pero para comprender lo que es la energía se entienden primero las leyes de Newton.

Hay una estrecha interconexión entre experimento y teoría en la estructuración de una teoría Física. Todo experimento presupone cierta teoría. Con la obtención de nuevos resultados experimentales, la formulación de nuevas definiciones operacionales y la formulación de hipótesis va creciendo la estructura de la teoría correspondiente. De esa manera, fueron elaboradas las principales leyes de la mecánica. Mediante la incorporación de nuevos conceptos, nociones y métodos Físico Matemáticos: mecánica analítica (sistemas conservativos, trabajos virtuales, coordenadas generalizadas, función potencial, espacio de configuración, principios variacionales, etc.) se crean leyes más generales que implican a las anteriores como casos particulares. Todo lo anterior, es sintetizado en lo que se ha llamado la mecánica clásica, siendo su principio más general el principio variacional de Hamilton. 


\subsection{Mecánica de los Sistemas de Partículas}

Temas: Mecánica del cuerpo rígido. Mecánica de sistemas elásticos. Mecánica de Líquidos. Mecánica estadística clásica.

Justificación: La mecánica newtoniana fue básica en la invención de mecanismos y máquinas que iniciaron y contribuyeron al desarrollo de la llamada revolución industrial. En esa época, se consideraba que todo conocimiento, para merecer la categoría de científico, debía expresarse en términos de las leyes de la mecánica newtoniana. De esa manera se trató de dar una explicación mecanicista a los fenómenos electromagnéticos, al calor, a las reacciones químicas, etc. Algunas de estas extrapolaciones contribuyeron a desarrollos artificiales que más adelante condujeron a contradicciones y a resultados discrepantes con los datos de la experimentación; por ejemplo, al postular la existencia del "éter", medio difundido en todo el espacio absoluto, a través del cual se propagaría la luz.

Sin embargo, la aplicación de la mecánica newtoniana conduce a resultados positivos, en la descripción entre otros problemas: (a) la mecánica del cuerpo rígido, modelo en el cual se considera que un objeto sólido, es tal que para el sistema de partículas que lo conforman, la distancia en el tiempo entre dos de ellas permanece constante. (b) La mecánica de líquidos, particularmente, en estado de reposo, o con flujo laminar. (c) Las oscilaciones y ondas mecánicas.

En el momento histórico de formulada la teoría corpuscular de la materia, no se conocía la existencia de átomos y moléculas, esta teoría considera que la materia está hecha de corpúsculos (partículas) perfectamente localizables sometidos a las leyes de la mecánica racional de Newton; el estado de cada corpúsculo viene definido en cada instante por su posición y su velocidad (o su impulso); es decir, en total seis variables dinámicas. En efecto, con esta hipótesis, las magnitudes macroscópicas aparecen como los valores medios de ciertas variables dinámicas de un sistema constituido por un gran número de grados de libertad; no se puede plantear la cuestión de resolver exactamente las ecuaciones de evolución de tal sistema; por lo tanto, se debe recurrir a métodos estadísticos de investigación. Así nace y se desarrolla una nueva disciplina, la mecánica estadística con la cual, conociendo hoy la existencia de átomos y moléculas, podemos desarrollar desde un punto de vista moderno y unificado la teoría cinética de gases, la termometría, la calorimetría y la termodinámica clásica.

\subsection{Física Experimental II}

Temas: Estudio cualitativo de algunos hechos del electromagnetismo y líneas equipotenciales. Electrólisis y mediciones de voltaje, intensidad y resistencia. Ley de Ohm, ley de Joule y transductores. Medida de campos magnéticos e Inducción electromagnética. Relajación exponencial y medición de corrientes y tensiones alternas. Oscilaciones libres y amortiguadas, resonancia. Superposición de oscilaciones y osciladores acoplados. Propagación de ondas. Óptica geométrica. Óptica física: Difracción e interferencia de ondas.

Justificación: Las medidas eléctricas, electrónicas y ópticas juegan un papel importante en la ciencia experimental y la técnica. El extraordinario poder y versatilidad de los dispositivos electrónicos, en consecuencia, su extensa aplicación, obliga a los estudiantes de ciencias e ingenierías a familiarizarse con la instrumentación electrónica. Un conocimiento fundamental de los circuitos y dispositivos electrónicos, suficientes para apreciar el funcionamiento y características de los diversos instrumentos electrónicos que deberá utilizar en su carrera profesional.

Este curso persigue presentar una síntesis del estudio de fenómenos electromagnéticos, ondulatorios y ópticos, con la práctica del empleo de 
instrumentos de medidas eléctricas y electrónicas: electrómetros, multímetros, medidores de RCL, generadores de señal, osciloscopios, entre otros.

\subsection{Electromagnetismo Clásico}

Temas: Panorámica del electromagnetismo clásico. Propiedades fundamentales de los campos escalares y vectoriales. Electrostática. Electrodinámica. Introducción a la Física Electrónica.

Justificación: El deseo de unificar las distintas ramas de la Física ha sido siempre una de las preocupaciones más persistente de los físicos; un ejemplo de ello es el establecimiento que la electricidad, el magnetismo y la luz son aspectos diferentes de una misma cosa conocida como campo electromagnético. El progreso decisivo lo consigue Maxwell en 1855 al plantear las ecuaciones fundamentales del electromagnetismo; basándose en estas ecuaciones, sugiere la existencia de ondas electromagnéticas, previsión confirmada ulteriormente de forma espectacular por el descubrimiento de ondas radioeléctricas por Hertz y asimila la onda luminosa a una onda electromagnética particular, realizando así la síntesis de la óptica y el electromagnetismo.

En la tabla 4 se presentan las ecuaciones de Maxwell en el vacío. Puede verse claramente que el planteamiento correcto de las leyes de la Física contienen algunas ideas poco familiares (campo, flujo, circulación, etc.), que va requiriendo

\begin{tabular}{|ll|}
\hline Forma Integral & Forma diferencial \\
$\oint \vec{E} \cdot \overrightarrow{d A}=\frac{Q_{\text {neta encerrada }}}{\varepsilon_{o}}$ & $\vec{\nabla} \cdot \vec{E}=\frac{\rho}{\varepsilon_{o}}, \vec{E}=-\vec{\nabla} V$ \\
$\oint \vec{B} \cdot \overrightarrow{d A}=0$ & $\vec{\nabla} \cdot \vec{B}=0$ \\
$\oint \vec{E} \cdot \overrightarrow{d l}=-\frac{\partial \Phi_{\vec{B}}}{\partial t}$ & $\vec{\nabla} \times \vec{E}=-\frac{\partial \vec{B}}{\partial t}$ \\
$\oint \vec{B} \cdot \overrightarrow{d l}=\mu_{o} I+\mu_{o} \varepsilon_{o} \frac{\partial \Phi_{\vec{E}}}{\partial t}$ & $\vec{\nabla} \times \vec{B}=\mu_{o} \vec{J}+\mu_{o} \varepsilon_{o} \frac{\partial \vec{E}}{\partial t}$ \\
\hline
\end{tabular}

Tabla 4. Ecuaciones de Maxwell en el vacío métodos Físico matemáticos más avanzados para su descripción. Por esta razón se necesita la dedicación de un tiempo de entrenamiento previo para entender lo que significan estas palabras.

\subsection{Ondas}

Temas: Dinámica de las oscilaciones. Osciladores acoplados y modos normales. Propagación de ondas. Fenómenos ondulatorios. Ondas de materia. Naturaleza de la luz. Óptica geométrica. Óptica física.

Justificación: Lo fundamentalmente interesante en este curso es el hecho de que muchos fenómenos físicos totalmente diferentes, y aparentemente no relacionados, pueden ser descritos en conjunto por una teoría Física coherente: La teoría de las Ondas. Con un formalismo similar, usando la misma terminología, se trata el tema unificado de oscilaciones mecánicas y electromagnéticas, ondas mecánicas, electromagnéticas y de materia y fundamentos de la óptica.

Las bases de datos revelan un gran número de registros sobre uno cualquiera de los fenómenos oscilatorios, ondulatorios y ópticos: la oscilación de un péndulo, las olas del mar, los colores del arco iris, la luz de las estrellas, el sonido que emite la cuerda tensa, la transmisión remota de programas de radio y televisión, las microondas, la holografía, la polarización de la luz, las ondas de la mecánica cuántica y muchos otros. Este curso brinda la oportunidad especial de desarrollar razonamientos de analogías entre los fenómenos mecánicos y los electromagnéticos y así examinar, por ejemplo, equivalencias entre los circuitos eléctricos y los sistemas mecánicos. En la tabla 5 se

\begin{tabular}{|c|c|c|}
\hline Ecuación de Onda & $\begin{array}{l}\text { Onda mecánica } \\
\qquad \frac{\partial^{2} y}{\partial x^{2}}=\frac{1}{v^{2}} \frac{\partial^{2} y}{\partial t^{2}}\end{array}$ & $\begin{array}{l}\text { Onda electromagnética } \\
\qquad \begin{array}{l}\frac{\partial^{2} E}{\partial t^{2}}=\frac{1}{\mu_{o} \epsilon_{\epsilon}} \frac{\partial^{2} E}{\partial x^{2}} \\
\frac{\partial^{2} B}{\partial t^{2}}=\frac{1}{\mu_{o} \epsilon_{o}} \frac{\partial^{2} B}{\partial x^{2}}\end{array}\end{array}$ \\
\hline \multicolumn{3}{|c|}{ Solución Onda armónica simple } \\
\hline
\end{tabular}

Tabla 5. Analogía entre fenómenos mecánicos y electromagnéticos 
ilustra la analogía entre fenómenos mecánicos y electromagnéticos reflejada en la expresión de su ecuación de onda y su correspondiente solución de onda armónica simple.

\subsection{Física Moderna}

Temas: Postulados de la relatividad. Postulados de la mecánica cuántica. Estructura de átomos, núcleos, sólidos, líquidos, gases y plasmas. Enlaces moleculares. Comportamientos térmicos de la materia, Transiciones de Fase. Propiedades de conducción de dieléctricos, semiconductores, conductores y superconductores. Magnetismo en átomos y sólidos. Estadística cuántica.

Justificación: Existen dos pilares fundamentales en los que se apoya la Física Moderna (Greene, 2002): Uno es la Relatividad General, que proporciona un marco teórico para la comprensión del universo a una escala máxima: estrellas, galaxias, cúmulos (o clusters) de galaxias, y aún más allá, hasta la inmensa expansión del propio universo. El otro pilar es la mecánica cuántica, que ofrece un marco teórico para la comprensión del universo a escalas mínimas: moléculas, átomos, y así hasta las partículas subatómicas, como los electrones y los quarks. A lo largo de los últimos años de investigación los físicos han confirmado experimentalmente, con una exactitud casi inimaginable, la práctica totalidad de las predicciones que hace cada una de estas teorías.

Desde un punto de vista histórico, las ideas cuánticas contribuyeron a una notable unificación de los conceptos de la Física fundamental al tratar las partículas materiales y la radiación en el mismo plano (Cohen-Tannoudji, Diu, \& Laloe, 1977). En el estado presente de nuestro conocimiento científico, somos cien por ciento conscientes que los objetos y hechos a muy pequeña escala, por ejemplo, átomos, enlaces químicos, propagación de electrones en un conductor, semiconductor o superconductor, propiedades magnéticas de los materiales, no pueden ser entendidos en términos de la mecánica Newtoniana, ellos sólo son entendidos en términos de la Física Cuántica.

\section{Gestión Educativa}

Debe considerarse que la reforma curricular es un proceso dinámico, que involucra no solo a estudiantes y profesores (directos implicados en el proceso enseñanza-aprendizaje) sino también a las directivas universitarias encargadas de organizar y normalizar estos cambios. Es necesario garantizar la intensidad horaria que permita el desarrollo pleno de los cursos programados, los espacios físicos de aulas de clase y laboratorios, equipamientos necesarios y organización de personal docente altamente cualificado y auxiliares responsables de sacar adelante la dinámica curricular.

Resulta de gran valor mencionar aquí la tesis, que sin duda tuvo notable éxito en la segunda posguerra, cuando se formaron las líneas directrices bajo las cuales se llevó a cabo la reconstrucción de Europa. El desarrollo científico es un bien al que ningún país puede renunciar, no solo por cuanto posee un valor en sí mismo, sino también porque es estímulo para el desarrollo tecnológico y económico. En cierto sentido, según esta tesis, el desarrollo científico implica automáticamente el desarrollo tecnológico y este último el económico (Cini \&Violini, 1985).

Precisamente, en Estados Unidos después de posguerra, dado el gran impulso que tuvo la Física, creciendo con una investigación cada vez más activa, con la promoción de universidades y fundaciones creadas para tales fines, fueron alentados un número sustancial de físicos que trabajaban en la vanguardia de la investigación para ofrecer sus contribuciones sobre ¿Qué? y ¿Cómo? exponer la Física a nivel superior de manera que tuviera en cuenta la formación que deberían alcanzar los futuros profesionales y reflejara la tremenda revolución que había sufrido la ciencia para estos nuevos tiempos. Fruto de 
éste trabajo resultaron los cursos: The Feynman Lectures on Physics (Feynman, Leighton, \& Sands, 1998), Berkeley Physics Course and Laboratory (BPC, 1973), Physical Science Study Committee (PSSC, 1964). En ese mismo sentido el M.I.T. (The Massachusetts Institute of Technology) ha patrocinado muy buenos cursos (M.I.T., 2014). Estos cursos marcan bien el camino. Son cursos para aprender Física y para aprender qué es la Física, cuál es el significado de la Física en la cultura de los tiempos actuales.

Gran parte de las discusiones públicas sobre si la ciencia y la técnica son buenas o malas, versan en realidad sobre el buen o mal uso que se hace de la tecnología que de ellas deriva. Caminar por la luna fue una hazaña de ingeniería aeroespacial mundial, posible gracias a dos siglos de Física clásica, la mayor parte de ella lograda por físicos que no tenían la menor idea de lo que era poner los pies sobre este satélite natural. Los múltiples, magníficos y utilísimos productos tecnológicos con que cuenta hoy la humanidad, las edificaciones, la radio, la televisión, las plantas de energía nuclear, los radioisótopos para el estudio de las enfermedades humanas, los vehículos espaciales teledirigidos, etc., simbolizan toda la gama de opciones que tiene la sociedad para la aplicación de la Física, son consecuencia de la acumulación de conocimientos de la Física de otras épocas y de las nuevas conquistas en el campo de la Física pura.

Cuando en el trabajo científico se aprende a entender y controlar una clase de evento particular, los fundamentos de estática-dinámica, el electromagnetismo clásico, las ondas radioeléctricas, se empieza a formar un grupo de especialistas cuyo trabajo consiste en hacer útil esta nueva adquisición. En esta forma, la ingeniería civil, la ingeniería de fluidos, la ingeniería aerodinámica, la ingeniería eléctrica, la ingeniería de radio, la ingeniería electrónica, la ingeniería nuclear, etc., nacen a partir de conocimientos científicos especiales, siendo estudiados con más dedicación formando una nueva especialidad técnica por el correspondiente grupo profesional de ingenieros. ¡Así ha avanzado la humanidad!

Si hay ejemplos históricos en que la invención práctica es anterior a la formulación de la correspondiente ley científica, es el caso de la palanca, la cual fue formulada por Arquímedes después de que los obreros venían utilizándola durante siglos. Sin embargo, es claro notar históricamente, que a medida que la ciencia y la técnica avanzan se hace cada vez menos probable que se produzca una invención sin el previo conocimiento de la ley o las leyes científicas que explican su funcionamiento.

La Física está permanentemente aportando nuevos conocimientos. Los cursos sugeridos aquí fundamentan para el desarrollo de cursos posteriores, cursos de pregrado, posgrado o formación avanzada; por ejemplo: Campos electromagnéticos, Métodos Físico matemáticos de solución a problemas electrodinámicos, Física electrónica (Física del estado sólido, Física de semiconductores, etc.), Física de Materiales, Métodos y Técnicas Experimentales de Análisis (análisis térmico, fluorescencia de rayos $\mathrm{x}$, difracción de rayos $x$, técnicas nucleares, etc.), Física de los Medios Granulares, etc., para ingenieros interesados en procurar desarrollar hoy o en el futuro, soluciones más eficaces y eficientes a los problemas técnicos.

\section{Conclusiones}

La Física aporta a la formación del ingeniero y a su desempeño profesional un conjunto amplio de conocimientos y habilidades. Durante su formación en los cursos de Física Básica el estudiante aprende a: ordenar y analizar los datos resultantes de la experimentación; crear hipótesis y definiciones operacionales sobre cuya base se puedan estructurar afirmaciones que sistematicen la descripción de los datos obtenidos; deducir conclusiones de dichas afirmaciones y confrontar sus conclusiones con 
los resultados experimentales. Conocer con propiedad, el desarrollo histórico, su contenido y sus métodos matemáticos, de las teorías físicas estudiadas y adquirir cierta habilidad operativa para aplicar las teorías estudiadas en la solución de situaciones particulares.

Reafirmamos dos ingredientes importantes para el proceso de enseñanza-aprendizaje de la Física. Primero, el desarrollo afectivo entre lo que el estudiante quiere aprender y lo que el maestro enseña. Segundo, el desarrollo de esa actitud de confianza ante lo que se realiza, de ese enamoramiento por lo que se hace. Al comprender resulta que ¡La física es más que divertida!.

\section{Referencias}

Berkely Physics Course [BPC]. (1973). Volumen I, II, III, IV yV. Reverté, S. A.

Cernuschi, F. (1981). Experimento, Razonamiento y Creación en Física. Washington, D. C.: Unión Panamericana.

Cini, M., \& Violini, G. (1983). Motivaciones para una política científica. Revista Naturaleza, educación y ciencia, No. 3. 29-32. Recuperado de: http:// www.cienciayjuego.com/Museo\%20de\%20la\%2 0Ciencia\%20y\%20el\%20Juego\%20-\%20Revista $\% 20$ Naturaleza\%203.pdf

Cohen-Tannoudji, C., Diu, B., \& Laloe, F. (1977). Quantum Mechanics. France: Hermann and by John Wiley \& Sons. Inc.
Freeland, H. (1984). La búsqueda de respuestas. Fondo Educativo Interamericano.

Feynman, R. P., Leighton R. B., \& Sands, M. (1998). Física, Volumen I, II y III. México: Maddison Wesley Longman de México S. A. de C.V.

Gamow, G. (1976). Gravedad. Argentina: Editorial Universitaria de Buenos Aires.

Greene, B. (2001). El universo elegante. Recuperado de: http://violetadedios.files. wordpress.com/2011/01/el-universo-elegantede-brian-greene.pdf

Hernández, C. A., López, J., \& Jaramillo A. (2006). El video argumental, herramienta en la enseñanza de las ciencias "El arte de la ciencia: Galileo Galilei". Recuperado de: http://repositorio.colciencias .gov.co/bitstream/11146/205/1/2\%20\%20.75Ma nerasGenerarConocimiento\%20\%202.pdf

Massachusetts Institute of Technology [MIT] (2014). Mitopencourseware. Recuperado de: http://ocw.mit.edu.

Physical Science Study Committee [PSSC]. (1964). Física. Editorial Bedout.

Sabino, C. (1992). El proceso de investigación. Bogotá D.C.:Panamericana

TryEngineering. (2014). Life of an Engineer. Recuperado de: http://www.tryengineering. org/explore-engineering/life-engineer 